\title{
The Functional Neuroanatomy of Tourette's Syndrome: An FDG PET Study III: Functional Coupling of Regional Cerebral Metabolic Rates
}

\author{
K.J. Jeffries, M.H.S., C. Schooler, Ph.D., C. Schoenbach, M.A., P. Herscovitch, M.D., T. N. Chase, \\ M.D., and A.R. Braun, M.D.*
}

Functional coupling of regional cerebral metabolic rates for glucose measured with [ $\left.{ }^{18} \mathrm{~F}\right]$-Fluoro-2-deoxy-D-glucose PET was compared in 18 drug-free patients with Tourette's Syndrome (TS) and 16 age- and sex-matched control subjects. Pearson product-moment correlation matrices containing correlations between metabolic rates in regions sampled throughout the brain were generated independently for TS patients and controls and compared. Significant differences between Z-transformed correlation coefficients were used to identify group differences, and revealed that the connectivity of the ventral striatum was most severely affected in TS. Changes in the coupling of other brain areas - primary motor areas, somatosensory association areas, and insula - also appeared to differentiate TS patients and controls. Evaluation of interrelationships between cortico-striato-thalamo-cortical circuits revealed the existence of functional connections between the motor and lateral orbitofrontal circuits in both groups, however, a reversal in the pattern of these interactions differentiated TS patients and controls. In controls, activity in these circuits appeared to be negatively correlated-i.e. increased activity in one is associated with relative inactivity the other. In TS patients, on the other hand, activity in the motor and lateral orbitofrontal circuits appears to be positively coupled. These results lend further credence to the hypothesis that altered limbic-motor interactions represent a pathophysiological hallmark of this disease.

[Neuropsychopharmacology 27:92-104, 2002]

(C) 2002 American College of Neuropsychopharmacology.

Published by Elsevier Science Inc.
KEY WORDS: Tourette syndrome; Movement disorder; Brain; Basal ganglia; Dopamine; Striatum

From the Language Section, Voice Speech and Language Branch, National Institute on Deafness and Other Communication Disorders, National Institutes of Health (KJJ, ARB), Section on SocioEnvironmental Studies, National Institute of Mental Health, NIH (CS, CS), PET Department, Clinical Center, NIH (PH), and Experimental Therapeutics Branch, NINDS, NIH (TNC).

Address correspondence to: A. R. Braun, M.D., Language Section, NIDCD, NIH, Building 10, Room 5N118A, Bethesda, Maryland, 20892, Tel.: (301) 402-1497, Fax: (301) 594-5274, E-mail: brauna@ nidcd.nih.gov

Received December 19, 2000; revised October 8, 2001; accepted October 25, 2001.

Online publication: 11/02/01 at www.acnp.org/citations/ Npp110201199.
Tourette's Syndrome (TS) is a poorly understood neurological disorder characterized by multiple motor and phonic tics and associated behavioral disturbances. While TS is clearly a disorder of motor function, the nature of the tics and the associated cognitive and behavioral features suggest that the pathogenesis extends beyond the motor domain. The motivational tension that commonly precedes motor and vocal tics and the sense of relief that follows their expression, as well as the irritability, impulsivity, depression or self-injurious behavior that may characterize more complex cases, all tend to suggest that brain regions associated with motivation or affect may also be involved. The generation of symptoms in TS might in fact reflect an abnormality of brain systems that effect neural transmission from the limbic 
to the motor systems-an abnormal coupling of brain regions involved in motivation and action.

We have previously reported (Braun et al. 1993, 1995) that TS may be characterized by lower metabolic rates in discrete portions of inferior limbic cortex and striatum, and in subcortical limbic structures, and higher metabolic rates in the superior sensorimotor cortices. However, brain regions do not operate in isolation; they function as elements in a series of neural networks distributed throughout the central nervous system, and it is likely that abnormal functional relationships between these regions play a role in the pathophysiology of TS. Positron emission tomography (PET) data can be analyzed using statistical methods that attempt to identify these regional interrelationships. That is, correlations between regional metabolic rates for glucose can be used to characterize networks of regions that appear to be affected in a neuropsychiatric disease, and, potentially, to pinpoint a breakdown of organized transynaptic activity which might distinguish the disorder (Clark et al. 1984; Horwitz et al. 1984, 1991).

The purpose of the present study is to determine how the functional interactions between brain regions are altered in TS-information that may shed light on system level abnormalities in this disease. We describe a 3-step analysis performed on $\left[{ }^{18} \mathrm{~F}\right]$-Fluoro-2-deoxy-D-glucose PET data acquired in TS patients and normal volunteers. The first stage constitutes a global analysis in which all interregional correlations between brain regions found to be significant in our first publication (Braun et al. 1993) are compared in order to identify those regions in which disordered functional interrelationships best characterize TS patients. The second step involves the generation and analysis of brain maps depicting interactions between specific brain regions and the rest of the brain-the specific brain regions were chosen based on the results of our previous work and their likelihood to play a role in TS. The third step involves the evaluation of functional interactions between elements of cortico-striato-thalamo-cortical (CSTC) circuits, initially described by Alexander et al. (1986), in order to identify potential sites at which abnormal limbicmotor interactions may occur.

\section{SUBJECTS AND METHODS}

\section{Subjects}

Eighteen patients with TS, sixteen males and two females aged $33 \pm 7$ years (mean $\pm S D$; range $23-49$ years; 16 right-handed, 2 left-handed) consented to participate in this study after being informed of its purposes, risks and potential benefits. Sixteen control subjects, eleven males and five females aged $34 \pm 10$ years (mean \pm SD; range $20-50$ years; 15 right-handed, 1 left-handed) gave informed consent prior to participation. The diagnosis of TS was confirmed by history and physical examination that excluded concurrent neurological, medical or unrelated psychiatric illnesses, and conformed to DSMIII-R criteria. All patients were drug free at the time of scanning. Four patients had received neuroleptics and two had received nonneuroleptic medications, all of which were withdrawn at least two weeks prior to the PET studies. Nine additional patients had a history of exposure to neuroleptic or nonneuroleptic drugs, but had not received treatment for at least one year prior to these studies. One patient had never been exposed to neuroactive medications.

In the second publication in this series (Braun et al. 1995) patients were categorized with respect to the historical presence or absence of six common behavioral features: obsessive-compulsive symptoms, echophenomena, coprolalia, impulsivity, self-injurious behavior, and depression. The number of behavioral features present was categorized into "high" = 3-6 symptoms ( $28 \%$ of the patients), "medium" $=2$ symptoms ( $39 \%$ of the patients), and "low" $=0-1$ symptom ( $33 \%$ of the patients).

Further details about the subjects are described in the first publication (Braun et al. 1993). The protocol under which the study was conducted (NIH 81-N-98) was reviewed and approved by the NIH/NINDS Institutional Review Board and the NIH Radiation Safety Committee.

\section{PET Scans and Image Analysis}

PET scans were performed as described previously (Braun et al. 1993). Five $\mathrm{mCi}$ of $\left[{ }^{18} \mathrm{~F}\right]$-Fluoro-2-deoxy-D-glucose (FDG), was injected intravenously over a period of one minute. Ten-minute scans were initiated $30-45 \mathrm{~min}$ after injection of FDG. Local cerebral metabolic rates for glucose (CMRglu) were calculated according to the method of Brooks (1982). Planes of section were selected for analysis and regions of interest (ROI) were placed to sample normalized CMRglu. Names and locations of all regions sampled were tabulated in the first publication (Braun et al. 1993). Regional CMRglu values were normalized using whole-brain metabolic averages. Several additional ROIs were used in this study that were not included in the first publication.

Regional metabolic rates were obtained by placing templates consisting of both circular and irregular ROI on PET planes of section. ROIs were independently translated and placed over the local maximum (activity peak). The irregular ROIs were applied in the cortex in order to maximize the use of anatomical detail there and to include the entire range of metabolic data. Cortical-cerebrospinal fluid (CSF) and cortical-white matter boundaries were thresholded interactively and the cortical mantle was divided using a radial template that could be adjusted with reference to the midline and ma- 
jor cortical landmarks. Regional perimeters were reduced by one pixel to decrease in-plane partial volume averaging and overlap between contiguous regions. These irregular cortical ROIs typically enclosed an area of $100-300 \mathrm{~mm}^{2}$.

Circular ROIs, ranging in diameter from 5.2 to $7.8 \mathrm{~mm}$ depending upon the size of the anatomic area sampled, were applied to subcortical structures and cortical regions (e.g., insula, medial temporal, and calcarine cortices) in which anatomic detail was less precise. Data reduction was performed in these instances by averaging these values for structures within an image plane in which more than one small circular ROI was applied. This process yielded 182 ROIs in all-91 in each hemisphere-an average of approximately 20 per slice. Templates were designed so that regions could be compared with coded areas in an atlas (Damasio and Damasio 1989) in which both functional and cytoarchitectonic descriptors are assigned to anatomical areas of interest.

\section{Statistical Analysis}

Global Correlational Analysis. Pearson product-moment correlation matrices for metabolic rates in all regions sampled $(\mathrm{n}=182)$ were generated independently for TS patients and controls (Winer 1971). The derivation of these matrices is illustrated schematically in Figure 1, upper panel. TS and control matrices were then compared parametrically in a series of steps illustrated schematically in Figure 1, lower panel. A Fisher Z-prime transformation (Fisher 1922) of the correlation coefficients was performed in order to generate a normal distribution of these values. Interregional correlations in TS and control matrices were then compared. Difference scores were calculated by subtracting individual transformed correlation coefficients derived for TS patients and controls. Difference scores are themselves Z-values for which probability levels can be calculated using a standardized normal distribution table. Difference Z-scores with an absolute value exceeding a threshold value of 1.96 were retained in the difference matrix and used to identify regional interrelationships which differentiated patients and controls. Comparison of the sums of the absolute values of the difference Z-scores for each region was used as a means of identifying regions in which altered coupling most robustly differentiated TS patients and controls.

Construction of Brain Maps. A line was placed on brain maps whenever the absolute value of the Z-transformed correlation coefficient between regions exceeded a threshold value of 1.96 (Figures 2-6). Lines that represent significant pairwise differences are marked with asterisks. In most cases a functional/anatomical category depicted in a brain map contained several discrete ROIs (from 1 to 6 ROIs). A line was placed on a brain map if the largest transformed correlation co-
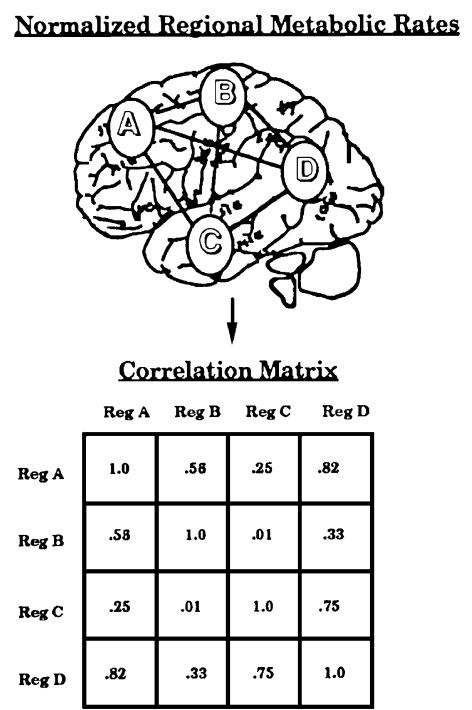

Proiection

Transformation

Subtraction

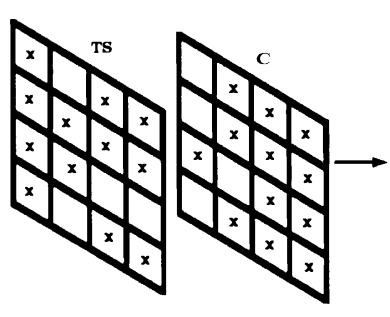

Transformed Matrices

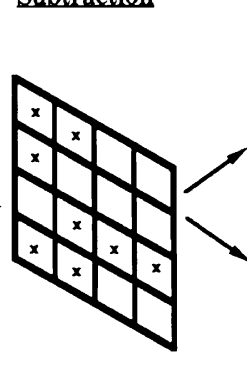

Difference Matrix

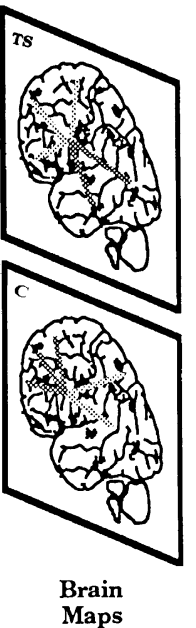

Figure 1. Schematic illustration of techniques used to analyze correlations between regional metabolic rates. Upper panel: Derivation of correlation matrices from regional PET data. Lower panel: Steps involved in the comparison of Tourette (TS) and control (C) matrices. In order to identify differences in regional coupling that distinguish patients from controls: (1) a Z-prime transformation of correlation coefficients is performed; (2) matrices are subtracted, generating difference scores; and (3) significant differences are represented by projecting the correlation coefficients back onto individual brain maps (see Methods section for details).

efficient for any ROI contained in the functional/anatomical category in either hemisphere exceeded threshold. When more than one of these ROIs had a transformed correlation coefficient exceeding threshold, the largest transformed coefficient was used. It should be noted that the threshold of 1.96 is not corrected for the multiple comparisons of ROI samples from which the highest value was chosen. 


\section{Ventral Striatum}

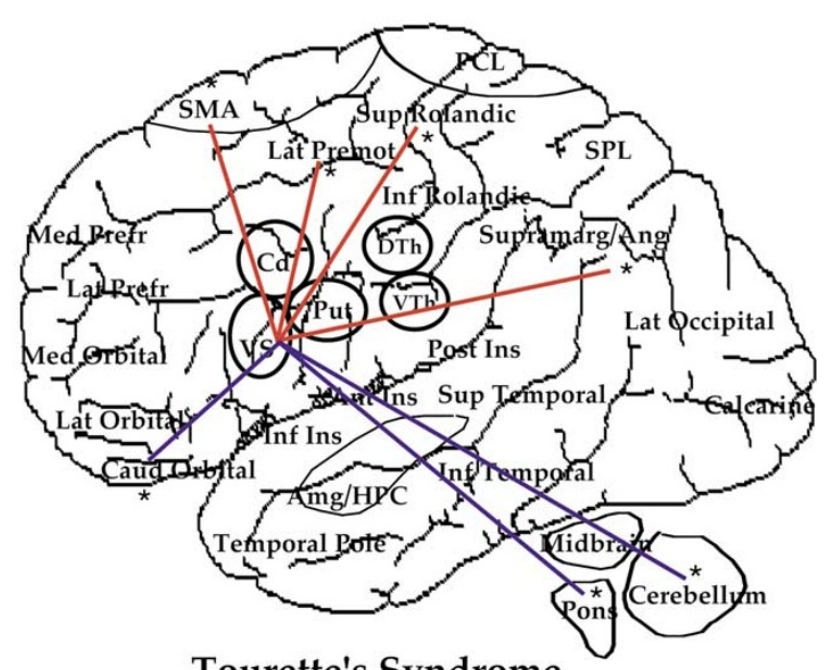

Tourette's Syndrome

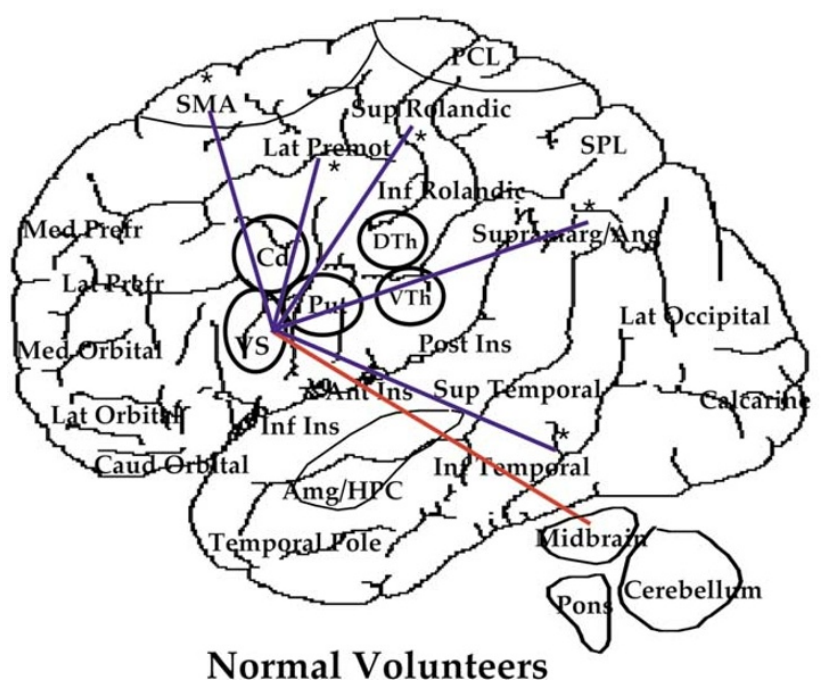

Figure 2. Brain map illustrating correlations between regional metabolic rates in Tourette's patients and controls. TS data are illustrated in the upper portion and normal volunteers in the lower portion. Functional associations of the ventral striatum are illustrated. Red indicates a positive correlation between brain regions, blue a negative correlation. Instances in which correlations significantly differentiate TS patients and controls, as well as instances in which significant correlations are present in both groups, are illustrated. Significant differences, indicated with an asterisk, represent instances in which a difference score $>1.96$ in absolute value was obtained and one or the other of the correlation coefficients exceeded threshold values $(p<.05$, not corrected for multiple comparisons). Congruencies are illustrated if correlations between regions in both groups exceeded threshold values. See Methods section for details.

\section{Putamen}

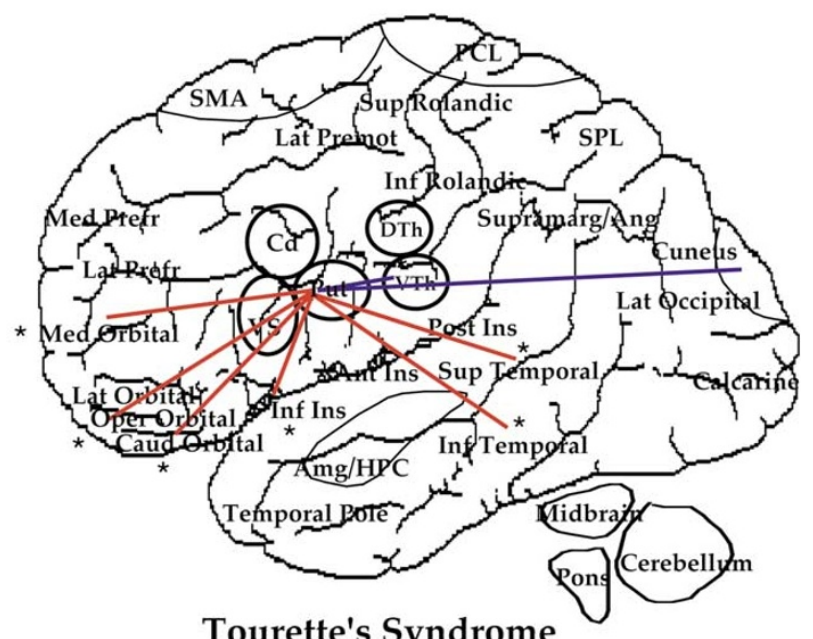

Tourette's Syndrome

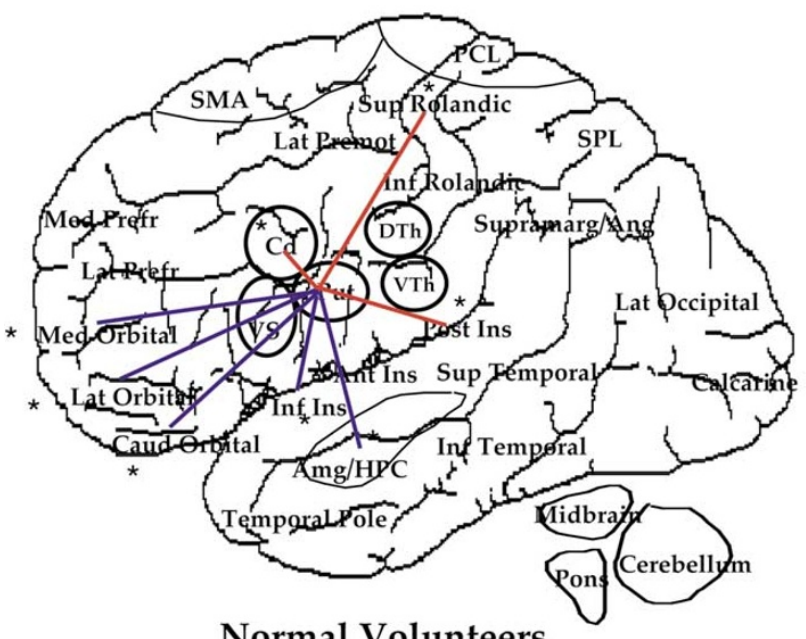

Figure 3. Brain map illustrating correlations between regional metabolic rates in Tourette's patients and controls. TS data are illustrated in the upper portion and normal volunteers in the lower portion. Functional associations of the putamen are illustrated. Please see Figure 2 legend for details.

Measurement Model. A measurement model (Joreskog and Sorbom 1996) was used in order to address the issues of highly correlated samples and error in the ROI measurements. Our model treats general functional brain areas as first order latent variables whose indicators are the multiple ROIs sampled from the PET scan data. The measurement model estimates activity of the component latent variables (the general brain areas) as measured by these observed variables (the ROI samples). In doing so, it provides estimates of the covariation of the latent constructs that take into account measurement error. Each general brain area is treated as a latent fac- 


\section{Superior Parietal Lobule}

\section{Anterior/Posterior Insula}
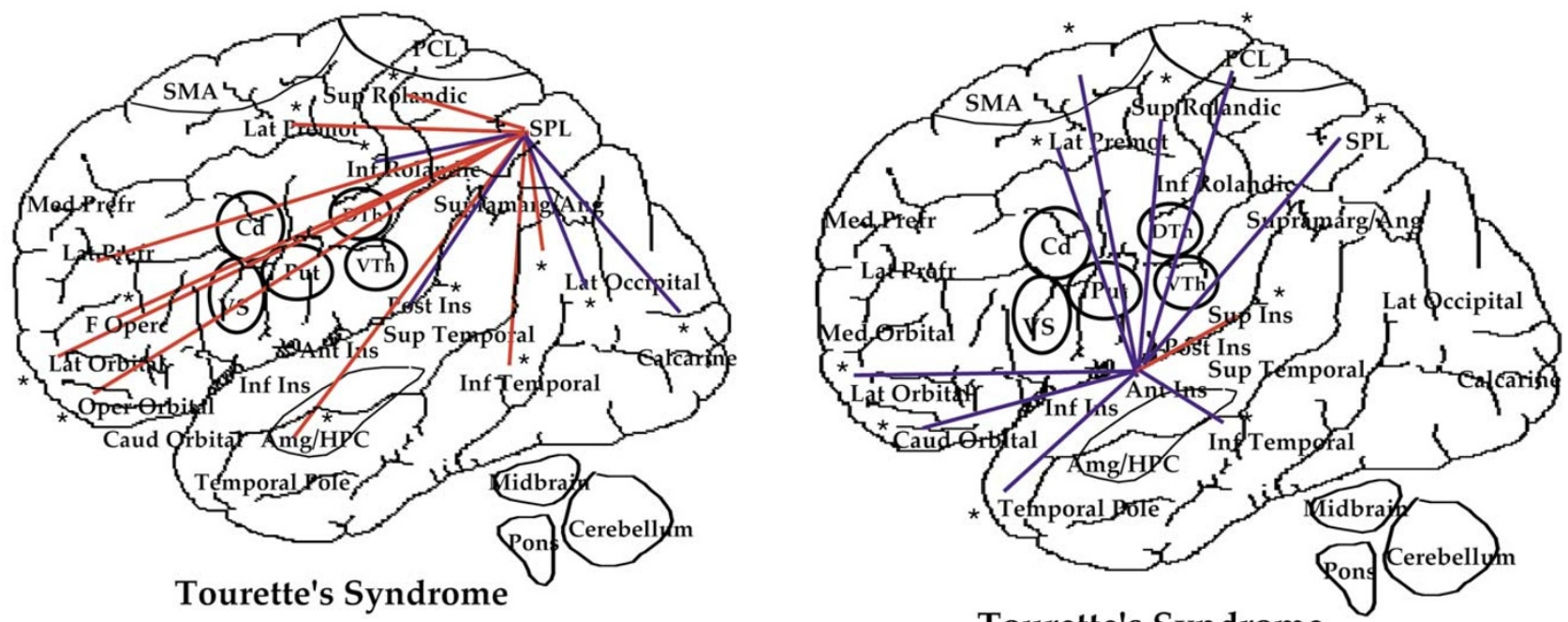

Tourette's Syndrome

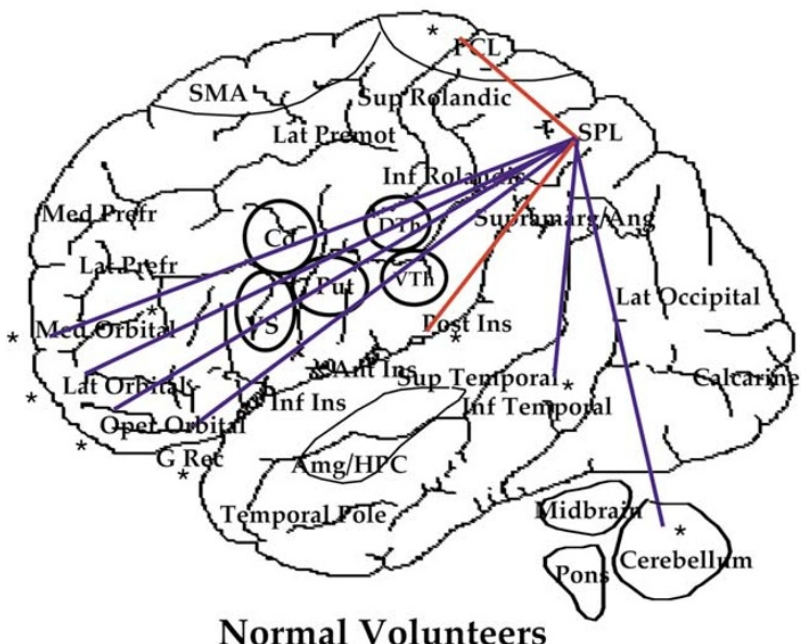

Figure 4. Brain map illustrating correlations between regional metabolic rates in Tourette's patients and controls. TS data are illustrated in the upper portion and normal volunteers in the lower portion. Functional associations of the superior parietal lobule are illustrated. Please see Figure 2 legend for details.

tor in a confirmatory factor analysis, and the activity of this latent variable is what is ultimately used as a refined and accurate measurement of activity. The psi matrix obtained from the measurement model analysis was used to provide the correlations between the latent factors, which were used as the principal measure of regional interaction in this report.

In our model, the general functional brain areas represent elements of two of the cortico striato-thalamo cortical (CSTC) circuits described by Alexander et al. (1986):

the motor circuit, which includes the putamen, ventral thalamus, and supplementary motor area; and the lateral orbitofrontal circuit, which includes the ventral striatum (ventromedial caudate), dorsal thalamus, and lateral orbital cortex (see Figure 7). Other areas that feed into these two circuits (e.g. Rolandic cortices, superior parietal lobule, and temporal cortex) were included in the model in order to account for some potential sources of additional variance. The measurement model was implemented us-

ing LISREL (version 8.02, Scientific Software Inc.).

Figure 5. Brain map illustrating correlations between regional metabolic rates in Tourette's patients and controls. TS data are illustrated in the upper portion and normal volunteers in the lower portion. Functional associations of the insula are illustrated. Please see Figure 2 legend for details. 


\section{Inferior Rolandic Cortex}
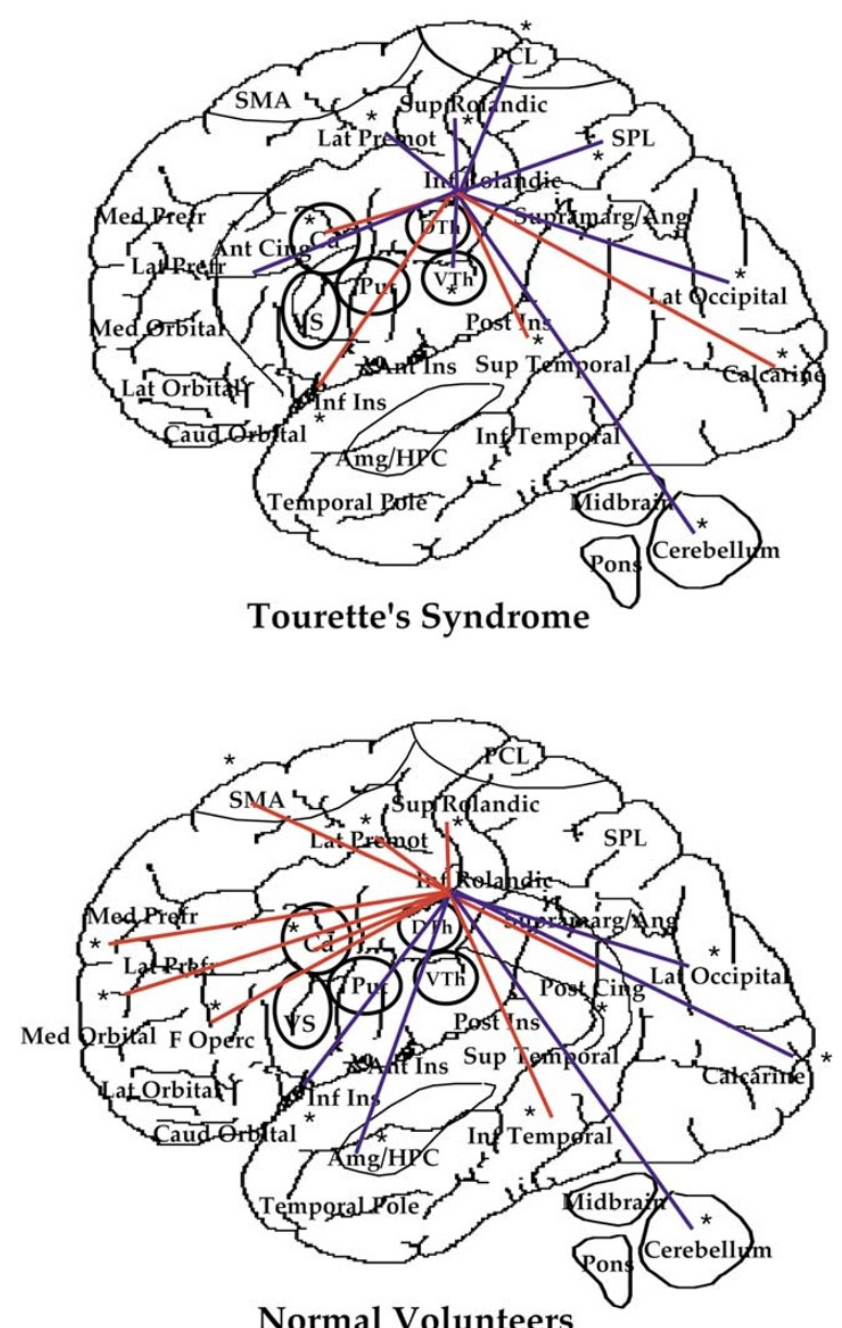

Figure 6. Brain map illustrating correlations between regional metabolic rates in Tourette's patients and controls. TS data are illustrated in the upper portion and normal volunteers in the lower portion. Functional associations of the inferior Rolandic cortex are illustrated. Please see Figure 2 legend for details.

\section{RESULTS}

\section{Global Correlational Analysis}

The global correlational analysis serves as a means of identifying regions in which altered coupling most robustly differentiates TS patients and controls. As noted in the methods section, the principal differences were determined by subtracting Z-transformed correlation coefficients derived for TS and control matrices, applying a threshold to the difference scores and summing the absolute values of these for each region.

The sum of absolute difference Z-scores was markedly higher for the ventral striatum (161.8) than for any other brain region and represented the only instance in which this value was greater than three standard deviations above the overall mean $(50.9 \pm 38.6) .34 .8 \%$ of all significant differences between TS and controls included the ventral striatum. Other regions which were significant in our first publication and in which these measures differentiated TS patients and controls (summed absolute difference Z-values greater than one standard deviation above the overall mean) are listed in Table 1.

\section{Brain Maps}

Brain maps depicting functional connections of ventral striatum, putamen, superior parietal lobule (SPL), insula, and inferior Rolandic cortex - regions that either our previous studies or the literature have suggested are likely to be involved in the pathogenesis of TS (Braun et al. 1993, 1995) - are illustrated in Figures 2-6. As described in the methods section, the illustrations depict significant correlations occurring in either the left or right hemispheres.

For this analysis we chose to use ventral striatum, putamen, SPL, insula, and inferior Rolandic cortex. From our earlier analysis of absolute CMRglu values in TS we know that ventral striatum, putamen, and inferior insula demonstrated decreased absolute CMRglu values in TS, while inferior Rolandic cortex demonstrated increased absolute CMRglu values in TS.

\section{Ventral Striatum}

The most striking changes involving the ventral striatum are observed in the reversal of functional relationships between this region of the striatum and motor cortices-lateral premotor cortex, supplementary motor area (SMA), and superior Rolandic cortex-in TS patients (Figure 2). In controls there is a consistent pattern of negative correlations between the ventral striatum and these motor areas. In TS patients, however, these correlations are uniformly positive.

Relationships between the ventral striatum and other subcortical structures are altered in TS patients as well. Metabolic activity in ventral striatum is negatively correlated with that in cerebellum, pons, and caudal orbital cortex, while correlations with these regions are absent in controls. Correlations between metabolic rates in ventral striatum and midbrain and inferior temporal cortex found in controls are not found in TS patients. There is a reversal from a positive correlation with supramarginal gyrus in TS patients to a negative correlation in controls.

\section{Putamen}

Metabolic activity in the putamen is negatively correlated with that in orbitofrontal cortices and the inferior regions of the insula in controls (Figure 3). In contrast, 

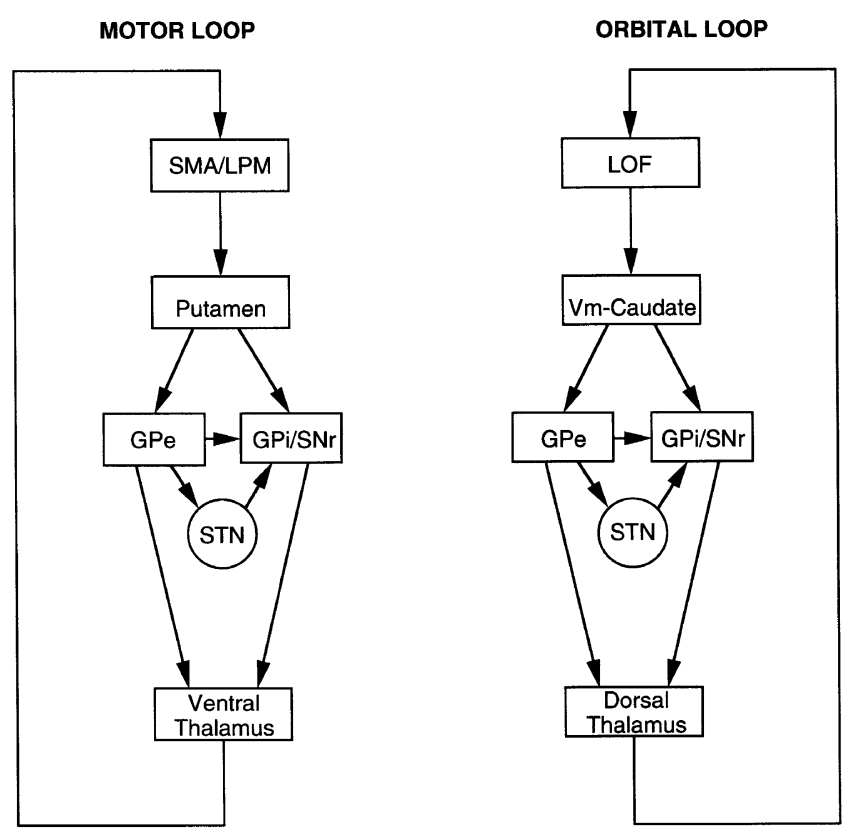

Figure 7. Simplified diagrams of motor and lateral orbitofrontal cortico-striato-thalamo-cortical circuits proposed by Alexander et. al. (1986, 1990). Each circuit engages specific regions of the cerebral cortex, striatum, pallidum (Gpi, Gpe), subthalamic nucleus (STN), substantia nigra (SNr), and thalamus. Pallidum and subthalamic nucleus were not included in the model because these regions are too difficult to resolve spatially in the PET scan. Abbreviations are as follows: Gpe: external segment of globus pallidus; Gpi: internal segment of globus pallidus; LOF: lateral orbitofrontal cortex; LPM: lateral premotor cortex; SMA: supplementary motor area; SNr: substantia nigra pars reticulata; STN: subthalamic nucleus; vm: ventromedial.

activity in the putamen and orbital and inferior insular regions is positively coupled in TS patients.

In addition, metabolic activity in the putamen is positively correlated with activity in the posterior insula in controls but not in TS patients, and the positive correlation between metabolic activity in putamen and superior Rolandic cortex which appears to characterize controls is absent in TS patients as well. A positive correlation with ventral thalamus and negative correlations with superior and inferior temporal cortices and superior cuneus occur only in TS patients. A positive correlation with the caudate nucleus and a negative correlation with the hippocampus occur only in controls.

\section{Superior Parietal Lobule (SPL)}

Functional connectivity of the SPL is illustrated in Figure 4 . In general negative correlations between activity in the SPL and other brain regions predominate in controls, while TS patients are characterized by a preponderance of positive correlations. For example, an array
Table 1. Cumulative absolute difference Z-scores of regions that demonstrated significant increases or decreases in CMRglu in TS (vs. control subjects) as reported in the first paper in this series (Braun et al. 1993) and had a cumulative absolute difference $Z$-score $>1 \mathrm{SD}$. These scores represent the sum of all Z-score differences between TS patients and controls that were greater than 1.96 in absolute value. Values in the SD column represent the number of standard deviations scores for each region fall above the mean of all cumulative absolute difference Z-scores. Asterisks denote significant increases or decreases in CMRglu in TS (vs. control subjects) as reported in the first paper in this series (Braun et al. 1993).

\begin{tabular}{lcc}
\hline Brain Region & $\begin{array}{c}\text { Cumulative } \\
\text { Z-score }\end{array}$ & SD \\
\hline Ventral Striatum & 161.8 & $3.2^{*}$ \\
Parrahippcampal Gyrus & 121.7 & $1.8^{*}$ \\
Superior Rolandic Cortex & 116.6 & $1.6^{* *}$ \\
Opercular Orbital Cortex & 105.5 & $1.2^{*}$ \\
Caudal Orbital Cortex & 102.4 & $1.1^{*}$ \\
\hline
\end{tabular}

CMRglu decreased in TS patients, ${ }^{* *} \mathrm{CMRglu}$ increased in TS patients

of negative correlations between SPL and orbitofrontal regions is found in controls, that is, increased activity in the orbitofrontal cortex is associated with decreased activity in the SPL, while correlations between SPL and orbital cortices are positive in TS patients. Positive correlations are also found-in TS patients but not in controls-between SPL and dorsolateral prefrontal, lateral premotor, and superior Rolandic cortices, as well as the frontal operculum and amygdala. TS patients and controls are further distinguished by different patterns of correlation between SPL and paracentral lobule, cerebellum, calcarine cortex, inferior Rolandic cortex, lateral occipital cortex, supramarginal gyrus, angular gyrus, inferior temporal cortex, and posterior insula. A negative correlation with the posterior insula and a positive correlation with the inferior temporal cortex in TS patients are both reversed in controls.

\section{Insula}

Functional relationships of the insula are illustrated in Figure 5. In almost every instance in which TS patients differ significantly from controls, correlations between activity in the insula and activity in other brain regions are negative in TS patients, and positive in controls. A predominant feature is a reversal in the pattern of correlations between insular regions and premotor and Rolandic areas. In contrast to controls, increased activity in the insula in TS patients is associated with decreasing activity in lateral premotor cortex, SMA, superior Rolandic cortex and paracentral lobule. Similarly, negative correlations between insular regions and orbital, paralimbic, parietal, and temporal cortices were observed in TS patients but were absent or reversed in controls. 
Positive correlations between activity in the insula and caudate, putamen, cerebellum, anterior cingulate cortex, hippocampus, and inferior insula were observed in controls, but not in TS patients. Negative correlations between activity in the insula and ventral striatum and lateral occipital cortex were observed in controls, but not in TS patients. In addition, correlations between anterior and superior regions of the insula itself were seen in both controls and patients. These were negative in controls, but positive in TS patients.

\section{Inferior Rolandic Cortex}

Functional relationships of the inferior portions of the primary motor cortex are illustrated in Figure 6. In general, control subjects are characterized by more positive, and TS patients by more negative, correlations between this region and other brain areas. Relationships between the inferior Rolandic cortex and premotor areas specifically differentiate TS patients and controls: patients are characterized by a negative correlation between inferior Rolandic and lateral premotor cortex, but this relationship is positive in control subjects-and in this case, involves both lateral premotor cortex and SMA. Functional coupling between inferior Rolandic and prefrontal areas (medial, lateral prefrontal, and frontal opercular cortices) observed in controls is absent in TS patients. Correlations between inferior Rolandic cortex and SPL, superior temporal cortex, ventral thalamus, anterior cingulate, and paracentral lobule occur only in TS patients.

Correlations between inferior Rolandic cortex and posterior cingulate, inferior temporal cortex, and amygdala occur only in controls. There is a reversal in correlation between TS patients and controls in inferior insula, calcarine cortex, and superior Rolandic cortex. There were both congruencies and differences in correlations with different parts of the cerebellum, caudate nucleus, and lateral occipital cortex.

\section{Cortico striato-thalamo cortical Circuits}

The measurement model applied to data from individual ROIs permitted generation of latent variables-estimates of activity in general brain areas that constitute elements of the lateral orbitofrontal and motor circuits (see Methods). Variables for lateral premotor cortex and SMA were combined into one "premotor" second-order latent variable due to colinearity of these variables. Variables for inferior Rolandic cortex and superior Rolandic cortex were combined into one "Rolandic" variable for the same reason.

A number of statistically significant differences between TS patients and controls were found in the correlations (derived as psi values in the measurement model) between elements of the circuits. These differences are illustrated in Figure 8.
The correlations between the cortical, striatal, and thalamic elements of the motor and lateral orbitofrontal circuits were examined (see Figure 8). Both groups demonstrated some significant correlations within the two CSTC circuits-i.e. between areas that are known to be anatomically connected. In both controls and TS patients, significant correlations were found in the motor circuit between putamen and ventral thalamus in both hemispheres and in the lateral orbitofrontal circuit between dorsal thalamus and lateral orbitofrontal cortex on the left. In addition, TS patients also demonstrated a significant correlation between right lateral orbitofrontal cortex and ventral striatum (Vm-caudate) in the lateral orbitofrontal circuit.

There were also unexpected connections between circuits in both TS patients and controls. In both groups, a bilateral pattern of significant correlations was observed between SMA (motor circuit) and ventral striatum (Vm-caudate, lateral orbitofrontal circuit) and between lateral orbitofrontal cortex (lateral orbitofrontal circuit) and putamen (motor circuit). Putamen and ventral striatum (motor and lateral orbitofrontal circuits, respectively) were significantly correlated in the right hemisphere of both groups.

In general, the pattern of correlations-both within and between the two circuits-differed significantly in TS patients and controls, and in each case, the correlations were reversed in sign.

In both hemispheres, the correlation between putamen and ventral thalamus (within the motor circuit) was positive in controls and negative in Tourette's patients. In the left hemisphere, the correlation between dorsal thalamus and lateral orbitofrontal cortex (within the orbitofrontal circuit) was positive in controls and negative in Tourette's patients.

The most dramatic reversals occur in correlations between the two circuits. Cross-circuit correlations between SMA (motor circuit) and ventral striatum (orbitofrontal circuit) and lateral orbitofrontal cortex (lateral orbitofrontal circuit) and putamen (motor circuit) were uniformly negative in controls, but positive in Tourette's patients. This pattern of reversal occurred in both hemispheres.

\section{DISCUSSION}

There were three salient findings in this study: First, the ventral striatum appears to be the most significantly affected brain region in TS. Second, changes in the functional connections of other brain areas-primary motor areas, somatosensory association areas, and insulaalso appear to distinguish TS patients and controls. In many instances these changes constitute a reversal of coupling between limbic and motor areas, and may account for some of the clinical features of TS. Third, TS 
LEFT HEMISPHERE

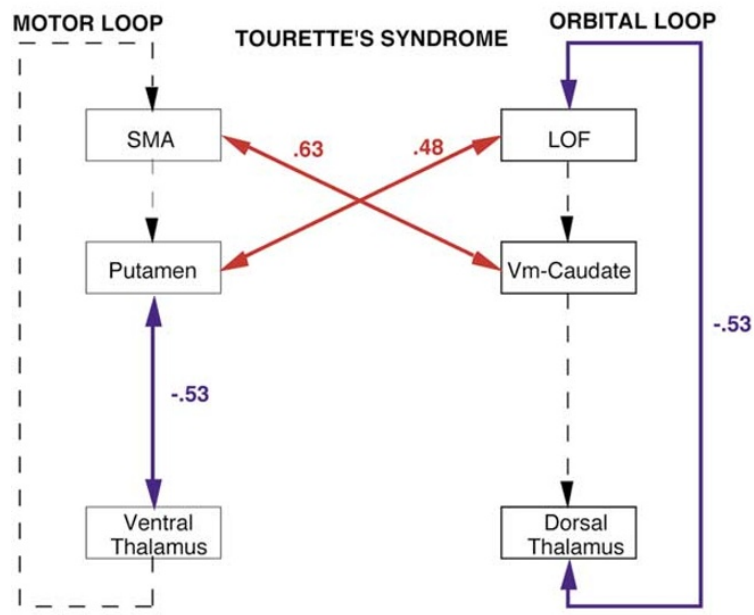

NORMAL VOLUNTEERS

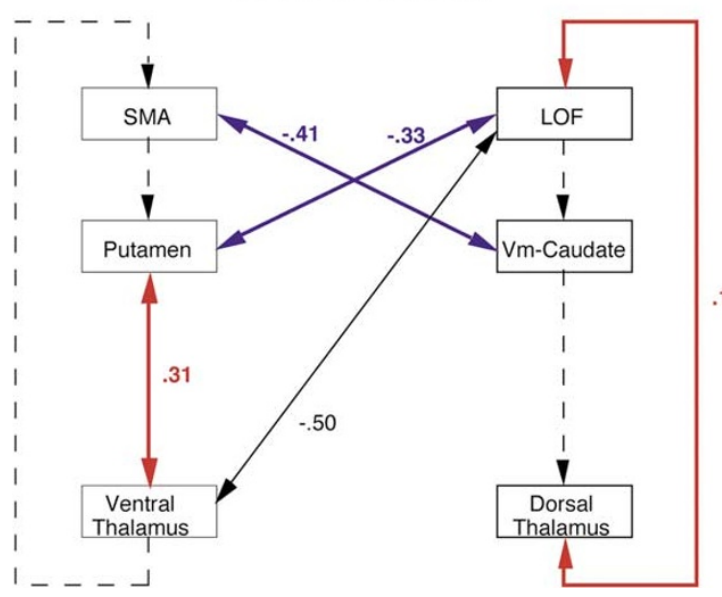

RIGHT HEMISPHERE

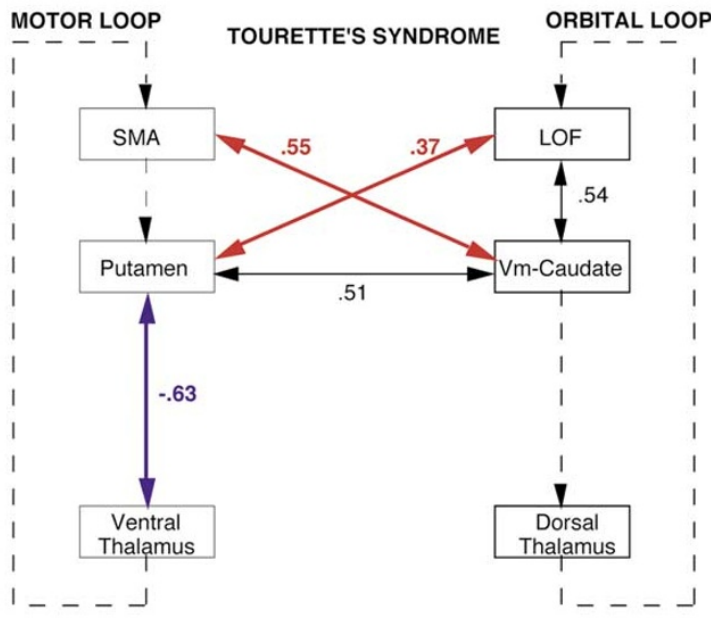

NORMAL VOLUNTEERS

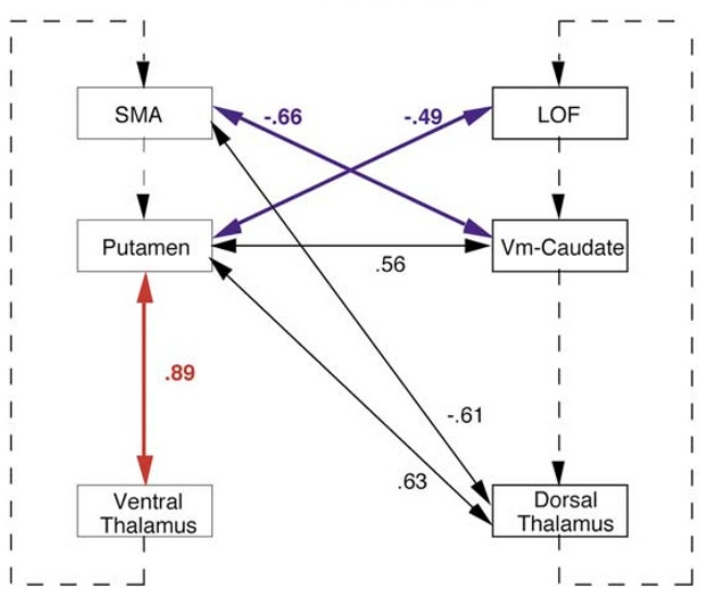

Figure 8. Interactions within and between motor and orbitofrontal cortico-striato-thalamo-cortical circuits. The circuits in this figure are simplified versions of Figure 7; the figures now also demonstrate the results of the SEM analysis. Note that the effects of pallidum and STN (as illustrated in Figure 7) cannot be measured directly because of the small size of the STN and the inability to resolve GPe and GPi. As a result, their combined effect is represented via a net correlation between striatum and thalamus. Bold correlation coefficients on color coded arrows indicate significant differences between TS and controls. Red arrows between regions indicate positive correlations, blue arrows negative correlations. Black arrows indicate other statistically significant correlations between regions that did not demonstrate a statistically significant difference between groups. Dashed lines represent the theoretical flow of information through the circuits; the solid lines indicate that a statistically significant correlation between the connected regions was observed.

may be specifically associated with abnormal connections between basal ganglia, thalamus, and cortexwithin the structure of the CSTC circuitry.

We did not correct for the multiple comparisons of interregional correlations because our aim was to find altered patterns of correlations between groups rather than the significance of any one particular difference in correlations. We considered it more important in this analysis to achieve a better balance between type I and type II error, in the interest of detecting any potentially important alterations in patterns of correlations. Because of the multiple comparisons, only the $r$ and Z-score val- ues are reported and no claims are made about the corresponding $\mathrm{p}$-values.

It should also be mentioned that the present findings could reflect metabolic changes related to inhibition of abnormal movements (Peterson et al. 1998). Vocal or motor tics were only rarely observed during the period of FDG uptake, and although patients were not explicitly instructed to suppress symptoms, suppression may have occurred without subjects' being consciously aware of it.

Additionally, studies of the natural history of TS demonstrate that tics generally diminish in severity 
during adolescence, and the persistence of severe tics into adulthood is relatively unusual. Imaging studies such as this one that include only clinically identified adults with TS are therefore unlikely to be representative of the total population of subjects who have a lifetime diagnosis of TS.

Comorbid obsessive-compulsive disorder (OCD) was present in 11 of the TS patients. We do not have data on the prevalence of attention deficit hyperactivity disorder in this group of patients. Brain abnormalities due to these conditions may have had some influence on our results. Relationships between OCD and regional cerebral metabolism were demonstrated in our second publication, but because of the number of patients, we were not able to take this into account in this analysis.

Similarly, there is some evidence to suggest that TS in female subjects may not be identical to TS in male subjects. Because there were only two female TS patients out of the 18 patients, it is not possible to discern if our results are in any way related to gender related manifestations of TS.

\section{Ventral Striatum}

Every one of the statistical measures applied in this study identified the ventral striatum as a region in which altered functional relationships most robustly discriminate TS patients and controls. The salient differences in patterns of connectivity of the ventral striatum (which was independently identified as a significant discriminator in our previous reports (Braun et al. 1993)) again suggest that this region plays a role in the pathogenesis of TS.

Glucose utilization in the ventral striatum was found to be reduced in TS patients in our first publication (Braun et al. 1993). The ventral striatum consists of the nucleus accumbens septi, the olfactory tubercle, and ventromedial parts of the caudate nucleus and putamen (Afifi and Bergman 1998). The region of interest designated the ventral striatum in this study should include metabolic information from each of these areas. The ventral striatum appears to play a role in motivation, especially in behaviors that are guided by the limbic system (Gilman et al. 1996), and may ultimately be involved in the regulation of movement by coupling limbic and motor mechanisms. The ventral striatum has been called the "limbic-motor interface" (Stevens 1973; Rolls and Williams 1987; Scheel-Kruger and Willner 1991), and is one of the principal sites in which information from limbic structures gains access to the motor system (Heimer et al. 1982; Mogenson 1987) in part via interactions with dorsal elements of the core nuclei of the basal ganglia (Haber et al. 1985, 1990).

Our results suggest that in TS, functional coupling between limbic and motor systems may be abnormal. In particular there appears to be a reversal of functional relationships between activity in the ventral striatum and cortical areas involved in the initiation of movement. In controls, activity in the ventral striatum is negatively correlated with activity in these motor regions. When the ventral striatum is metabolically active, the motor areas are relatively silent. In TS patients, however, these correlations are uniformly positive. Ventral striatum and motor cortices are all active-or activated-concurrently. These data suggest that the illness may be characterized, in part, by a distorted relationship between this striatal region and cortical areas involved in motor control. This is discussed further in the context of CSTC circuitry below.

\section{Putamen}

Glucose utilization in the putamen was found to be reduced in TS patients in our first publication (Braun et al. 1993). As observed with the ventral striatum, functional connectivity of the putamen also suggests abnormal coupling between limbic and motor systems in TS. In this case, there appears to be a reversal of functional relationships between activity in the putamen and cortical areas with a rich input from paleocortical limbic and paralimbic regions of the brain-orbitofrontal cortices and the related, inferior regions of the insula. Metabolic activity in the putamen is negatively correlated with these regions in controls. Activity in the putamen is, on the other hand, positively coupled with activity in orbitofrontal and inferior insular cortices in TS patients. This is discussed further in the section on CSTC circuitry below.

\section{Superior Parietal Lobule (SPL)}

The SPL was selected for analysis because it appears to be involved in complex integrative functions related to the organization and initiation of movement. This region is necessary for the generation of movement-related potentials in humans (Knight et al. 1989) and therefore, along with the SMA and prefrontal cortices to which it is connected, may be intimately involved in the preparation for and initiation of motor activity (Goldberg 1985).

Coupling between the SPL and motor as well as limbic regions was very different in TS patients and controls, and these altered relationships could play a role in the pathophysiology of TS. Stronger coupling between the SPL and motor regions in TS patients and an overall reversal in its coupling with limbic regions-positive in TS patients and negative in controls (Figure 4) suggest that functional connectivity of this posterior parietal area may mediate abnormal limbic-motor interactions in this disorder.

In this context, the role of the SPL as a somatosensory association area is also of potential relevance to 
premonitory sensory urges-unpleasant sensory perceptions or mental awarenesses that precede tic behaviors that are momentarily relieved by the performance of the tics (Leckman et al. 1997). Abnormal connections between the SPL, motor, and limbic regions could underlie this feature of the illness as well.

\section{Anterior and Posterior Insula}

Glucose utilization in the insular cortex was found to be reduced in TS patients in our first publication (Braun et al. 1993). Other imaging studies (Weeks et al. 1996) have implicated the insula in TS as well. The potential importance of the anterior insula in TS stems from its extensive interconnections with the orbitofrontal areas which may play a role in behavioral inhibition (Iversen and Mishkin 1970; Passingham 1972; Fuster 1997).

In the insula, like in the ventral striatum and SPL, the most striking changes in TS include altered functional relationships with motor regions-lateral premotor cortices, SMA, superior Rolandic cortex, and paracentral lobule.

\section{Primary Motor Cortices}

Primary motor cortex constitutes the final common pathway for execution of movement. Glucose utilization in the primary motor cortex was found to be increased in TS patients in our first publication (Braun et al. 1993). The inferior portions of this area are associated with movements of the upper extremities, face, and vocal apparatus (lips, tongue, larynx) - areas which represent the predominant anatomical distribution of tics in TS (Leckman and Cohen 1999). Activity of the primary motor cortex is regulated by regions at higher levels-specifically by premotor areas, which organize and initiate complex sequences of movements, and are themselves regulated by projections from other brain regions. There are two major premotor systems: a medial system centered upon the SMA, which may organize self initiated movements, and the lateral premotor system, which may organize movements expressed in response to exteroceptive stimuli. Relationships between primary motor cortex and each of these premotor areas appear to be fundamentally altered in TS.

As in the insula, SPL, putamen, and ventral striatum, interregional correlations between primary motor cortex and other brain regions that differentiate TS patients and controls appear to be reversed in sign. In addition, activity in the primary motor cortex is positively correlated with that in the medial and lateral prefrontal, and frontal opercular cortices in controls. No significant correlations between these regions are detected in TS patients. This suggests a change in communication between primary motor areas and areas related to both selective and exclusionary attention, and possibly lan- guage related functions as well. A functional uncoupling of inferior Rolandic and frontal opercular regions may provide a substrate for dysregulation of orolaryngeal movements and the production of vocal tics.

\section{Cortico-striato-thalamo-cortical Circuits}

A number of our results can be interpreted in the context of the cortico-striato-thalamo-cortical (CSTC) circuitry proposed by Alexander et al. (1986). Alexander initially described five segregated pathways connecting basal ganglia, thalamus, and frontal cortex. Information was thought to be progressively integrated as it traversed these pathways, which were designated the motor, oculomotor, dorsolateral prefrontal, lateral orbitofrontal, and anterior cingulate circuits (Alexander et al. 1986). These were described as being segregated, organized in parallel, with only a minor degree of overlap, although more recently it has been suggested that there may be more communication between the circuits than originally proposed (Groenewegen et al. 1990, 1994; Joel and Weiner 1994, 1997; Parent et al. 2000).

Two recent reports have examined the potential involvement of CSTC circuitry in TS. Eidelberg et al. (1997) applied the Scaled Subprofile Model to FDG PET data in order to identify specific patterns of regional metabolic covariation associated with TS. This analysis identified two TS-related brain networks that were considered to correspond with the motor and limbic circuits. This study concluded that the metabolic landscape of TS is characterized by a nonspecific pattern of increased motor cortical activity and a reduction in the activity of limbic CSTC projection systems (Eidelberg et al. 1997).

Leckman et al. (1997) reviewed the evidence implicating CSTC circuits in the pathobiology of TS. The motor and anterior cingulate circuits were posited as the most likely circuits to be involved in TS, but the lateral orbitofrontal circuit was considered to be more important in the pathobiology of obsessive-compulsive disorder (Baxter et al. 1992; Insel 1992; Leckman et al. 1997).

We chose to examine the relationship between motor and orbitofrontal circuits on the basis of results of our previous analyses (Braun et al. 1993, 1995) implicating sensorimotor and ventral limbic circuits in orbitofrontal cortex and insula. Other CSTC circuits-such as the anterior cingulate circuit-were not examined in this study, although the results here obviously do not rule out similar abnormalities of interactions within and between elements of these circuits.

While we observed correlations between elements of the two circuits we evaluated, we also observed what appeared to be rather extensive functional interactions between the two circuits, in both control subjects and TS patients. These findings are consistent with more recent suggestions that there may be functional commu- 
nication between the circuits at certain points (Groenewegen et al. 1990, 1994; Joel and Weiner 1994, 1997; Parent et al. 2000).

In the present study, the existence of functional "crosstalk"-i.e. the frequent instances in which activity in elements of the different circuits is statistically coupled-suggests that information may be shared between motor and lateral orbital frontal circuits in both controls and TS patients. But the nature of this "crosstalk" differs dramatically in patients and controls, suggesting that activity in the circuits is segregated in controls by means of the circuits being reciprocally related. On the other hand the concurrent activity of the two circuits in TS patients suggests that activity is somehow abnormally integrated in TS.

Overall, these results support the notion that cortical and subcortical motor areas may be more readily influenced by limbic structures in TS patients than in controls. Essential features of the disorder-the association of tics with motivational tension, impulsivity, obsessions and compulsions that are often experienced as alien or due to the operation of a "second will" - might be explained in part by an abnormal positive coupling between motor cortices and limbic portions of the striatum and between motor portions of the striatum and orbital cortices.

The existence of functional interaction between the two CSTC circuits raises the question of what anatomical mechanism might facilitate it. Functional "crosstalk" could occur as a result of convergence between circuits proposed to occur at a number of levels-mediated via cortico-cortical connections, through overlap of dendritic fields within the striatum (Groenewegen et al. 1990, 1994), or convergence within outflow nuclei of the basal ganglia (Haber et al. 1995). Axonal collateralization in the basal ganglia has recently been pointed out as an important mechanism for interactions between circuits (Parent et al. 2000). Joel and Weiner (1994) have suggested a mechanism of between-circuit interaction by introducing the concept of split circuitry, which essentially results in two pathways projecting back to the frontal cortex via the thalamus.

A direct exchange of information between the two circuits is not the only feasible interpretation of the correlated activity between them. It is also possible that such correlations might occur as the result of an external factor with a divergent influence upon both circuits. For example, the mesencephalic dopamine projections to the striatum could play such a role.

Indeed, the clinical effects of dopamine agonist and antagonist agents in TS suggest that dopaminergic modulation of striatum may be abnormal in this disorder (Cummings and Frankel 1985). Such an abnormality would not be inconsistent with the perturbed interaction of the motor and associative circuits we observed. The mesolimbic dopamine system may serve a gating function at the level of the ventral striatum, regulating the flow of information from limbic structures to the pallidum, thereby governing response initiation (Yang and Mogenson 1984). The involvement of the ventral striatum in motivation and the fact that it appears to be functioning abnormally in TS provides a model for the pathogenesis of this disorder. If, due to alterations in dopaminergic modulation, impulses are no longer gated properly at the point of the ventral striatum, they might make their way-via interactions with premotor structures-directly into action. The results of this study are consistent with previous studies that have implicated the motor CSTC circuit in TS (Eidelberg et al. 1997; Leckman et al. 1997). The apparent altered functional interactions between limbic and motor structures we observed suggest that TS may be related to abnormal coupling of limbic/paralimbic and motor mechanisms. Further studies will be necessary in order to clarify the specific loci of abnormal limbicmotor coupling in this disease.

\section{REFERENCES}

Afifi AK, Bergman RA (1998): Functional Neuroanatomy: Text and Atlas. New York, McGraw-Hill Health Professions Division

Alexander GE, DeLong MR, Strick PL (1986): Parallel organization of functionally segregated circuits linking basal ganglia and cortex. Annu Rev Neurosci 9:357-381

Baxter LR Jr, Schwartz JM, Bergman KS, Szuba MP, Guze BH, Mazziotta JC, Alazraki A, Selin CE, Ferng HK, Munford P. (1992): Caudate glucose metabolic rate changes with both drug and behavior therapy for obsessive-compulsive disorder. Arch Gen Psychiatry 49:681-689

Braun AR, Randolph C, Stoetter B, Mohr E, Cox C, Vladar K, Sexton R, Carson RE, Herscovitch P, Chase TN (1995): The functional neuroanatomy of Tourette's syndrome: an FDG-PET Study. II: Relationships between regional cerebral metabolism and associated behavioral and cognitive features of the illness. Neuropsychopharmacology 13:151-168

Braun AR, Stoetter B, Randolph C, Hsiao JK, Vladar K, Gernert J, Carson RE, Herscovitch P, Chase TN (1993): The functional neuroanatomy of Tourette's syndrome: an FDG-PET study. I. Regional changes in cerebral glucose metabolism differentiating patients and controls. Neuropsychopharmacology 9:277-291

Brooks RA (1982): Alternate formula for glucose utilization using labeled deoxyglucose. J Nucl Med 23:538-539

Clark CM, Kessler R, Buchsbaum MS, Margolin RA, Holcomb HH (1984): Correlational methods for determining regional coupling of cerebral glucose metabolism: a pilot study. Biol Psychiatry 19:663-678

Cummings JL, Frankel M (1985): Gilles de la Tourette syndrome and the neurological basis of obsessions and compulsions. Biol Psychiatry 20:117-126

Damasio H, Damasio AR (1989): Lesion analysis in neuropsychology. New York, Oxford University Press 
Eidelberg D, Moeller JR, Antonini A, Kazumata K, Dhawan V, Budman C, Feigin A (1997): The metabolic anatomy of Tourette's syndrome. Neurology 48:927-934

Fisher RA (1922): On the mathematical foundations of theoretical statistics. Philos Trans R Soc Lond [A] 222:309-368

Fuster JM (1997): The prefrontal cortex: anatomy, physiology, and neuropsychology of the frontal lobe. Philadelphia, Lippincott-Raven

Gilman S, Newman SW, Manter JT, Gatz AJ (1996): Manter and Gatz's essentials of clinical neuroanatomy and neurophysiology. Philadelphia, F.A. Davis

Goldberg G (1985): Supplementary motor area structure and function: Review and hypotheses. Behav Brain Sci 8:567-616

Groenewegen HJ, Berendse HW, Wolters JG, Lohman AHM (1990): The anatomical relationship of the prefrontal cortex with the striatopallidal system, the thalamus and the amygdala: evidence for parallel organization. In Uylings HBM, Van Eden CG, De Bruin JPC, Corner MA, Feenstra MGP (eds), Progress in Brain Research. New York, Elsevier Science Publishers B.V. (Biomedical Division), pp 95-118

Groenewegen HJ, Berendse HW, Wouterlood FG (1994): Organization of the projections from the ventral striatopallidal system to ventral mesencephalic dopaminergic neurons in the rat. In Percheron G, McKenzie JS, Férger J, International Basal Ganglia Society. Symposium, The Basal Ganglia IV: New Ideas and Data on Structure and Function. New York, Plenum Press. pp 81-93

Haber SN, Groenewegen HJ, Grove EA, Nauta WJ (1985): Efferent connections of the ventral pallidum: evidence of a dual striato pallidofugal pathway. J Comp Neurol 235:322-335

Haber SN, Kunishio K, Mizobuchi M, Lynd-Balta E (1995): The orbital and medial prefrontal circuit through the primate basal ganglia. J Neurosci 15:4851-4867

Haber SN, Lynd EL, Mitchell SJA (1990): Comparison between dorsolateral and ventromedial striatal pathways through the monkey basal ganglia. Soc Neurosci Abstr 16:954

Heimer L, Switzer RD, Van Hoesen GW (1982): Ventral striatum and ventral pallidum. Components of the motor system. Trends Neurosci 5:83-87

Horwitz B, Duara R, Rapoport SI (1984): Intercorrelations of glucose metabolic rates between brain regions: application to healthy males in a state of reduced sensory input. J Cereb Blood Flow Metab 4:484-499

Horwitz B, Swedo SE, Grady CL, Pietrini P, Schapiro MB, Rapoport JL, Rapoport SI (1991): Cerebral metabolic pattern in obsessive-compulsive disorder: altered intercorrelations between regional rates of glucose utilization. Psychiatry Res 40:221-237

Insel TR (1992): Toward a neuroanatomy of obsessive-compulsive disorder [see comments]. Arch Gen Psychiatry 49:739-744

Iversen SD, Mishkin M (1970): Perseverative interference in monkeys following selective lesions of the inferior prefrontal convexity. Exp Brain Res 11:376-386

Joel D, Weiner I (1994): The organization of the basal ganglia-thalamocortical circuits: open interconnected rather than closed segregated. Neuroscience 63:363-379

Joel D, Weiner I (1997): The connections of the primate subthalamic nucleus: indirect pathways and the open-interconnected scheme of basal ganglia-thalamocortical circuitry. Brain Res Brain Res Rev 23: 62-78

Joreskog KG, Sorbom D (1996): LISREL8: User's Reference Guide. Chicago, Scientific Software International, Inc.

Knight RT, Singh J, Woods DL (1989): Pre-movement parietal lobe input to human sensorimotor cortex. Brain Res 498:190-194

Leckman JF, Cohen DJ (1999): Tourette's syndrome-tics, obsessions, compulsions: developmental psychopathology and clinical care. New York, John Wiley \& Sons

Leckman JF, Peterson BS, Anderson GM, Arnsten AF, Pauls DL, Cohen DJ (1997): Pathogenesis of Tourette's syndrome. J Child Psychol Psychiatry 38:119-142

Mogenson GJ (1987): Limbic-motor integration. Prog Psychobiol Psychol 12:117-170

Parent A, Sato F, Wu Y, Gauthier J, Levesque M, Parent M (2000): Organization of the basal ganglia: the importance of axonal collateralization. Trends Neurosci 23(10 Suppl): S20-27

Passingham RE (1972): Visual discrimination learning after selective prefrontal ablations in monkeys (Macaca mulatta). Neuropsychologia 10:27-39

Peterson BS, Skudlarski P, Anderson AW, Zhang $\mathrm{H}$, Gatenby JC, Lacadie CM, Leckman JF, Gore JC (1998): A functional magnetic resonance imaging study of tic suppression in Tourette syndrome. Arch Gen Psychiatry 55:326-333

Rolls ET, Williams GV (1987): Sensory and movement related neuronal activity in different regions of the striatum of the primate. In Schneider JS, Lidsky TI (eds), Sensory Considerations for Basal Ganglia Function. New York, Haber

Scheel-Kruger J, Willner P (1991): The mesolimbic system: principles of operation. In Willner $\mathrm{P}$, Scheel-Krüger J (eds), The Mesolimbic Dopamine System: from Motivation to Action (European Behavioural Pharmacology Society). New York, Wiley

Stevens JR (1973): An anatomy of schizophrenia? Arch Gen Psychiatry 29:177-189

Weeks RA, Turjanski N, Brooks DJ (1996): Tourette's syndrome: a disorder of cingulate and orbitofrontal function? QJM 89:401-408

Winer BJ (1971): Statistical Principles in Experimental Design. New York, McGraw-Hill

Yang CR, Mogenson GJ (1984): Electrophysiological responses of neurones in the nucleus accumbens to hippocampal stimulation and the attenuation of the excitatory responses by the mesolimbic dopaminergic system. Brain Res 324:69-84 